20

\title{
A SIMPLE MODEL FOR CELL LOSS PROBABILITY EVALUATION IN AN ATM MULTIPLEXER
}

\author{
R. Bolla, F. Davoli, M. Marchese
}

Department of Communications, Computer and Systems Science (DIST)

University of Genoa

Via Opera Pia, 13 - 16145 Genova, Italy

lelus,franco,dama@dist.unige.it

\begin{abstract}
The importance of the evaluation of the cell loss probability in an ATM multiplexer has constantly increased and has become one of the most investigated problems in this research area. The cell loss probability, in fact, is a quantity extensively used for CAC algorithms and resource allocation schemes in an ATM-based network, and the accuracy of the analytical evaluation can be very important to obtain satisfactory results concerning the control mechanism.

In this paper a particular model (namely, the Interrupted Bernoulli Process - IBP), which has been chosen for its simplicity, analytical tractability and also for being well suited for many common traffic types, is analysed and used to evaluate the cell loss probability in an ATM multiplexer. The traffic is considered to be divided into classes. Each traffic class is identified by parameters like peak, average bandwidth and average burst length; each source is modelled as an IBP. The superposition of the traffic sources belonging to a specific traffic class enters a dedicated buffer, whose service rate is kept constant for each class.

The results obtained by using the proposed model are compared with several simulation results, obtained by using the superposition of sources modelled with a Markov Modulated Deterministic Process (MMDP) to approximate the actual cell arrival process. The comparisons have been performed for different offered load situations and for different buffer lengths. A satisfactory accuracy is shown for most of the cases considered.
\end{abstract}

ATM, CAC, traffic source modelling.

Keywords

OIFIP 1997. Published by Chapman \& Hall 


\section{INTRODUCTION}

Call Admission Control (CAC), Traffic Shaping, Policing, Routing and other topics related to traffic control or monitoring in ATM networks have received a great deal of attention in the literature; the possibility of guaranteeing performance requirements to each user or to a certain class of users is both a very interesting challenge for the scientific community and a practical possibility for the future administrator pricing procedures. Thus, the decision about the acceptance of a new call entering the network (CAC), the monitoring and control of the user traffic, the Quality of Service (QoS) metrics, the allocation of network resources like bandwidth or buffer space are topics which have been extensively treated (Roberts, 1991, Saito, 1994, Onvural, 1994), along with more technological problems like ATM switching (Hui, 1990). In this context, the evaluation of the cell loss probability at an ATM buffer is a topical subject: this quantity has a very strong impact on CAC strategies, bandwidth allocation algorithms and on the performance evaluation of a multiservice network (Roberts, 1991, Onvural, 1994, Ross, 1995).

The evaluation problem may be considered as composed by two components: i) the choice of a mathematical model to describe the arrival process of the cells (see (Roberts, 1991), for a survey and (D'Ambrosio, 1995), for an analysis of the traffic behaviour); ii) the actual computation of the cell loss probability (Nagarajan, 1991, Chao, 1995, Baiocchi, 1993, Choudhury, 1995).

Concerning the first point, it is a subject widely treated for any type of traffic: from the characterisation of voice and data superposition (Sriram, 1986, Heffes, 1986, Cheng, 1996), to LAN traffic (Gagnaire, 1995), and video, which is the most complex one (Herrmann, 1995), since its behaviour depends on the type of pictures (motion or static), on the compression algorithm and also on the current filmed scene. In general, a complete separate analysis is needed in each case and a general purpose model is very difficult to obtain. In more detail, a complete introduction to the issues involved in asynchronous transfer of video along with an overview of video coding, rate, error and loss control can be found in (Karlsson, 1995). As already said, the description of the video traffic heavily depends on the service. Concerning video-conference, for example, the traffic is accurately described by a multistate Markov chain that can be derived from three traffic parameters like mean, correlation and variance (Elwalid, 1995). Otherwise, sequences with frequent scene changes such as entertainment television, news and sports broadcasts often need more complex models (Heyman, 1996). Modelling a generic video source is often so difficult that adaptive algorithms are used to implement congestion control schemes. For example, a real-time modulation of the source rate of a video encoder is provided in (Kanakia, 1995), while a real-time mechanism aimed at maximising the quality of service perceived by the users is shown in (Bolla, 1995). In (Lee, 1994), a method of traffic smoothing for frames of different video sources is proposed.

However, the superposition of Talkspurt-Silence sources (discrete version of the Markov Modulated Deterministic Process - MMDP) is regarded in the literature as a model well suited for some types of traffic (Cosmas, 1994) like voice, interactive data (low, average and high speed), retrieval of still pictures, LAN interconnection 
and CAD traffic. This model is often taken as a reference for the evaluation and testing of some approximations (Yang, 1995, Sohraby, 1993).

The Talkspurt-Silence model is considered to be the reference used in the simulations also in this paper. The analytical results obtained by using the IBP model, which is simpler to manage for analytical treatments are compared with the values obtained by using a simulator, whose traffic generator is the superposition of several sources, each one modelled with the Talkspurt-Silence.

The paper initially describes the Talkspurt-Silence and the IBP models in Section 2. In Section 3, the treated scenario is shown and an evaluation scheme approximating, in certain conditions, the cell loss probability is introduced along with a method already presented in the literature (Kamitake, 1989, Bolla, 1994), and used as a comparison in Section 4 where simulation results are shown. Section 5 contains the conclusions.

\section{TWO MODELS FOR A BURSTY SOURCE}

A single source that generates cells following the Talkspurt-Silence model alternates between phases of activity (called bursts) and periods of silence. The sojourn times and transitions between the two phases are governed by geometric distributions with known mean value. During the silence phase no cell is generated. Within the burst, cells are generated at constant intervals of time. A connection of a specific traffic class, say $h$, has a peak bandwidth $P^{(h)}$ [bits/s], and the channel where the call is routed has a transfer capacity of $C$ [bits/s]. $C$ is supposed to be an integer multiple of $\mathrm{P}^{(\mathrm{h})}$ to simplify the presentation, but this assumption does not affect the general concept. The period of time between two cell generations, indicated by $M^{(h)}$ [slots], in the following, is the ratio between the channel capacity and the peak bandwidth, i.e., $M^{(h)}=C / P^{(h)}$; in fact, time is assumed slotted and a cell can be considered only at slot boundaries. There are $\left(M^{(h)}-1\right)$ empty cells between two consecutive cell generations.

This behaviour can be simply modelled with an $\left(\mathrm{M}^{(\mathrm{h})}+1\right)$-state Markov chain, as shown in Figure 1.

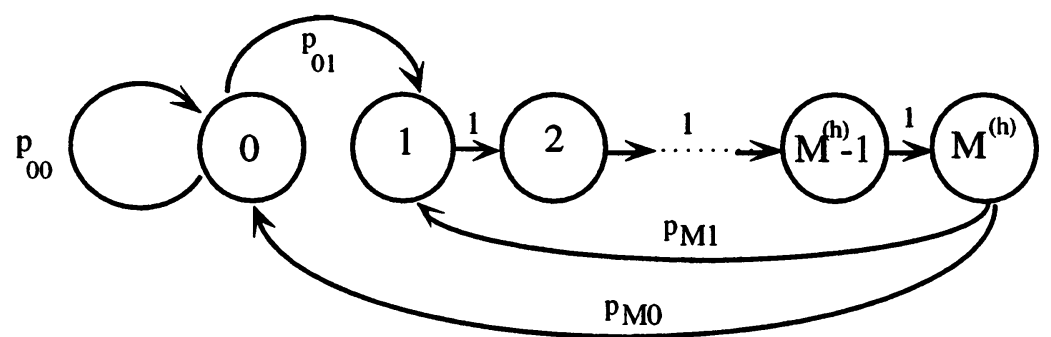

Figure $1\left(\mathrm{M}^{(\mathrm{h})}+1\right)$-state source model. 
State 0 is the Silence state, while states 1 through $M^{(h)}$ are bursty ones, but only 1 is a generating state. It is easy to compute the transition probabilities, obtaining:

$$
\mathrm{p}_{00}=1-\mathrm{p}_{01} ; \mathrm{p}_{01}=\frac{1}{\mathrm{~B}^{(\mathrm{h})}\left(\mathrm{b}^{(\mathrm{h})}-1\right)} ; \mathrm{p}_{\mathrm{M} 0}=\frac{\mathrm{M}^{(\mathrm{h})}}{\mathrm{B}^{(\mathrm{h})}} ; \mathrm{p}_{\mathrm{M} 1}=\frac{\mathrm{B}^{(\mathrm{h})}-\mathrm{M}^{(\mathrm{h})}}{\mathrm{B}^{(\mathrm{h})}}
$$

where $B^{(h)}$ is the average burst length for each connection of the generic traffic class $(h)$ and $b^{(h)}$ is the burstiness, defined as the ratio between the peak and average bandwidth $\left(D^{(h)}\right)$.

By computing the distribution and the density of the interarrival process, the result obtained is the discrete-time case of the density shown in (Sriram, 1986). But, to fully utilise the accuracy of these descriptions, the use of queuing theory and related approximations is needed to get the solution of complex mathematical problems. For example, a possible approach is based on the use of the characteristic function, as in (Sohraby, 1993). However, the description of the process resulting from the superposition of a certain number of Talkspurt-Silence connections is very difficult to obtain and its complexity probably too high to be used in an analytical treatment to evaluate the cell loss probability in an ATM multiplexer, which is the object of this paper. The superposition of Talkspurt-Silence calls has been utilised to get the simulation results in Section 5 , as already stated.

The model described and used in the following is an Interrupted Bernoulli Process (IBP), discrete-time version of the Interrupted Poisson Process (IPP) (Cosmas, 1994, Heffes, 1986). Also the IBP alternates periods of generation and phases of silence, where no cells are generated. The two periods are modulated by a geometric distribution, as in the previous case. The difference between the two models is that, during the activity phases, the IBP does not generate cells at fixed periodic time instants, but the emission is governed by a Bernoulli process. In Figure 2, the Markov chain which can simply model the behaviour of an IBP is shown: considering a channel with capacity $\mathrm{C}$, a bursty connection of a particular traffic class $\mathrm{h}$ can be described by a two-state model: 'active' and 'idle'.

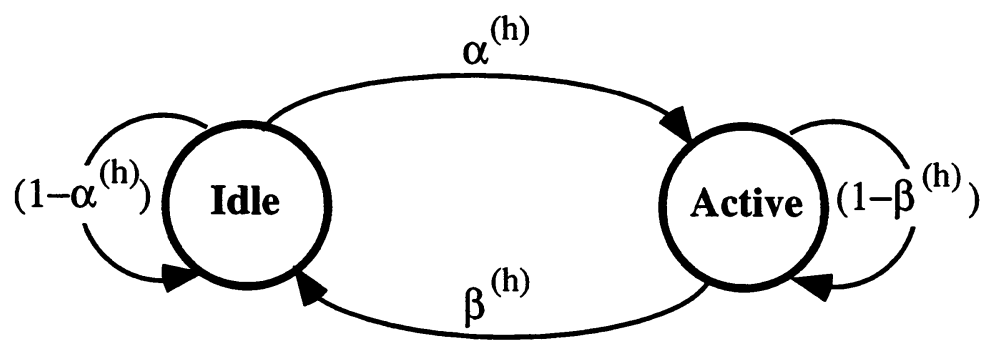

Figure 2 IBP bursty source model. 
Again, $\mathrm{P}^{(\mathrm{h})}$ being the peak bandwidth, $\mathrm{B}^{(\mathrm{h})}$ the mean value of the burst length (in cells) and $b^{(h)}$ the value of the burstiness, the probabilities to be in the 'idle' and 'active' state, are, respectively:

$\omega_{i}^{(h)}=\frac{\beta^{(h)}}{\alpha^{(h)}+\beta^{(h)}}=\frac{b^{(h)}-1}{b^{(h)}}$

$\omega_{\mathrm{a}}^{(\mathrm{h})}=\frac{\alpha^{(\mathrm{h})}}{\alpha^{(\mathrm{h})}+\beta^{(\mathrm{h})}}=\frac{1}{\mathrm{~b}^{(\mathrm{h})}}$

with $\alpha^{(h)}=\frac{1}{B^{(h)}\left(b^{(h)}-1\right)}$ and $\beta^{(h)}=\frac{1}{B^{(h)}}$. It is important to note that (2) and (3) are independent of the burst length.

As already stated, within the active state, the arrival process of cells is modelled by a Bernoulli process: at each discrete time instant, there is a cell arrival with probability $\Gamma^{(\mathrm{h})}=\frac{1}{\mathrm{M}^{(\mathrm{h})}}$, with $\mathrm{M}^{(\mathrm{h})}=\mathrm{C} / \mathrm{P}^{(\mathrm{h})}$ It is simple to demonstrate that the average number of empty cells between two successive cell generations for traffic class $(h)$ is $\left(M^{(h)}-1\right)$; in the Talkspurt-Silence model, where the distance between cells within a talkspurt is deterministic, $\left(M^{(h)}-1\right)$ is actually the number of empty cells between two successive cell generations. The IBP is an ON/OFF process where Bernoulli arrivals occur during a geometrically distributed period of time, with another geometrically distributed period of time where no cell generation occurs. It belongs to the class of renewal processes and it is the discrete time version of a two state MMPP.

In this paper, one single source is modelled with an IBP process and all sources belonging to a specific traffic class are supposed to be independent and identically distributed.

So, the stationary probability of having $n$ active connections out of $N^{(h)}$ accepted connections of the traffic class (h) can be written as

$$
v_{n}^{N^{(h)}}=\left(\begin{array}{l}
N^{(h)} \\
n
\end{array}\right)\left(\omega_{a}^{(h)}\right)^{n}\left(\omega_{i}^{(h)}\right)^{N^{(h)}-n}
$$

The probability $f_{j}^{(h)}(n)$ of having $j$ connections of traffic class (h) generating a cell, with $\mathrm{n}$ connections of the same class in the active state is: 


$$
f_{j}^{(h)}(n)= \begin{cases}0 & j<0 \\
\left(\begin{array}{l}
n \\
j
\end{array}\right)\left(\Gamma^{(h)}\right)^{j}\left(1-\Gamma^{(h)}\right)^{n-j} & 0 \leq j \leq n\end{cases}
$$

with

$$
\mathrm{n} \Gamma^{(\mathrm{h})}=\sum_{\mathrm{j}=0}^{\mathrm{n}} \mathrm{jf_{j }}(\mathrm{h})(\mathrm{n})
$$

where $\Gamma^{(\mathrm{h})}$ is the parameter of the Bernoulli distribution previously defined. After defining the model adopted and some important quantities which will be used in the following, the scenario of utilisation and a possible evaluation of the cell loss probability is presented in the next Section.

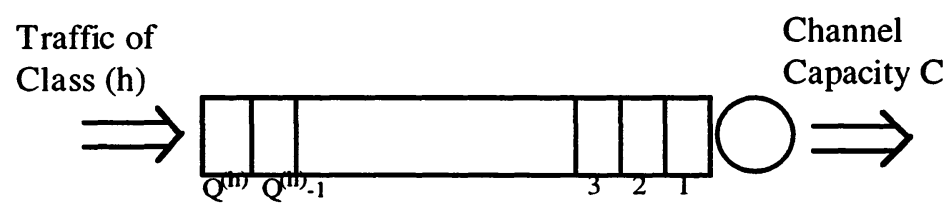

Figure 3 Buffer dedicated to the traffic class (h).

\section{EVALUATION OF THE CELL LOSS PROBABILITY}

In this paper the traffic is considered divided into classes, and each of them has a dedicated buffer. Due to the complete separation among the traffic classes, the situation can be sketched as in Figure 3: the multiplexed traffic of class (h), whose connections are supposed to be described by the IBP bursty source model introduced in Section 2, with each source independent of the others, enters a buffer of fixed length $Q^{(h)}$. The buffer is served by a single link with capacity $C$, which is completely dedicated to the traffic class (h). This approach, called "Service Separation" in (Ross, 1995), greatly simplifies the multiplexer analysis with respect to the complete statistical multiplexing of all the services (i.e., not dividing the buffer among the traffic classes) and does not give significant performance losses when services have greatly different QoS requirements or cell generation properties, as remarked in (Ross, 1995, p. 141).

Before introducing the two methods to evaluate the cell loss probability, the first one already presented in (Kamitake, 1989, Bolla, 1994), and the second one which is introduced in this paper, some quantities have to be defined. 
Let $G^{(h)}\left(n^{(h)}\right)$ be the transition matrix, whose elements $g_{i j}^{(h)}\left(n^{(h)}, Q^{(h)}\right)$ represent the probability of transition from the state where i cells are queued in the k-th slot to the state where $j$ cells are queued in the $(k+1)$-th slot, given $n^{(h)}$ connections in the active state. The computation of $g_{i j}^{(h)}\left(n^{(h)}, Q^{(h)}\right)$ is done by using the AF (Arrivals First) policy to model the process of simultaneous arrivals and departures in the queue (Gravey, 1992).

$$
\begin{aligned}
& g_{i j}^{(h)}\left(n^{(h)}, Q^{(h)}\right)= \begin{cases}0 & j<i-1 \\
f_{j-i+1}^{(h)}\left(n^{(h)}\right) & i \leq j<Q^{(h)}-1 \\
\sum_{s=Q^{(h)}-i}^{n^{(h)}} f^{(h)}\left(n^{(h)}\right) & j=Q^{(h)}-1\end{cases} \\
& g_{0 j}^{(h)}\left(n^{(h)}, Q^{(h)}\right)= \begin{cases}f_{0}^{(h)}\left(n^{(h)}\right)+f_{1}^{(h)}\left(n^{(h)}\right) & j=0 \\
f_{j+1}^{(h)}\left(n^{(h)}\right) & 0<j<Q^{(h)}-1 \\
n^{(h)} f_{S}^{(h)}\left(n^{(h)}\right) & j=Q^{(h)}-1 \\
s=Q^{(h)}\end{cases}
\end{aligned}
$$

$$
(\underset{a}{\text { with }} \underset{\mathbf{a}}{\mathbf{b}} \equiv 0 \text { if } \mathbf{a}<\mathbf{b})
$$

The quantities $\pi_{i}^{(h)}\left(n^{(h)}\right)$ represent the steady state probabilities of having $i$ cells inside the buffer and are computed by using the transition matrix $G^{(h)}\left(n^{(h)}\right)$. The steady state probability distribution

$$
\Pi^{(h)}\left(n^{(h)}\right)=\left[\pi_{0}^{(h)}\left(n^{(h)}\right), \pi_{1}^{(h)}\left(n^{(h)}\right), \ldots, \pi_{Q-1}^{(h)}\left(n^{(h)}\right)\right]
$$

of the queue length can be obtained by solving the following set of linear equations (note that, since the transitions occur just after beginning of service, $\pi_{Q}^{(h)}\left(n^{(h)}\right)=$ 0): 


$$
\begin{aligned}
& \Pi^{(h)}\left(n^{(h)}\right)=\Pi^{(h)}\left(n^{(h)}\right) G^{(h)}\left(n^{(h)}\right) \\
& \sum_{i=0}^{Q-1} \pi_{i}^{(h)}\left(n^{(h)}\right)=1
\end{aligned}
$$

The first method is referenced as 'method A' in the next Section.

If $n^{(h)}$ is the number of connections in the active state, the value of the cell loss probability can be computed as:

$$
P_{\text {loss }}^{(h)}\left(n^{(h)}, Q^{(h)}\right)=\sum_{i=0}^{Q^{(h)}-1} \frac{\sum_{j=0}^{(h)} \max \left(i+j-Q^{(h)}, 0\right) f_{j}^{(h)}\left(n^{(h)}\right)}{n^{(h)} \Gamma^{(h)}} \pi_{i}^{(h)}\left(n^{(h)}\right)
$$

where each quantity has been defined in the previous and in the current Section and $\max (a, 0)=\left\{\begin{array}{lll}a & \text { if } & a>0 \\ 0 & \text { if } & a \leq 0\end{array}\right.$.

The numerator in (12) represents the average number of lost cells in a slot, while the denominator is the average number of arrived cells (6). The ratio of these two quantities represents the rate of lost cells during a slot time, given a fixed buffer dimension, and it is averaged over the length of the buffer as well. Actually, it is a ratio of averages (or a rate) and it will approximate the probability that an arriving cell during a time slot finds the buffer full given the number $\mathrm{n}^{(\mathrm{h})}$ of connections in the active state.

The second approach which, as already said, is introduced in this paper, provides a quantity related to the above one, i.e., the cell loss probability given the number $\mathrm{n}^{(\mathrm{h})}$ of connections in the active state; however, the two quantities can give similar results only under some specific conditions. This evaluation is named 'method B' in the next Section.

In formula

$$
\mathrm{P}_{\operatorname{loss}}^{(\mathrm{h})}\left(\mathrm{n}^{(\mathrm{h})}, \mathrm{Q}^{(\mathrm{h})}\right)=\sum_{\mathrm{i}=0}^{\mathrm{Q}^{(\mathrm{h})}} \pi_{\mathrm{i}}^{(\mathrm{h})}\left(\mathrm{n}^{(\mathrm{h})}\right)\left(1-\sum_{j=0}^{\mathrm{Q}^{(\mathrm{h})}-\mathrm{i}} \mathrm{f}_{j}\left(\mathrm{n}^{(\mathrm{h})}\right)\right)
$$

Even in this case the quantities appearing in (13) have been already defined.

The quantity $\left(1-\sum_{j=0}^{Q^{(h)}-i} f_{j}(n(h))\right)$, which is also averaged over the buffer length, is the probability that there is at least one cell lost during a time slot given a fixed buffer dimension. This quantity would represent the probability of finding the buffer full in a slot (corresponding to the probability that an arriving cell during a time slot 
finds the buffer full, since the cell arrivals are independent of buffer state) if only one cell caould arrive in a slot, i.e., whenever the probability of having multiple arrivals in one slot would be null or negligible.

The aim, however, is to compute the cell loss probability $\overline{\mathrm{P}}_{\text {loss }}^{(h)}\left(N^{(h)}, Q^{(h)}\right)$, given $\mathrm{N}^{(\mathrm{h})}$ connections in progress of the traffic class (h). It can be evaluated as

$\bar{P}_{\operatorname{loss}}^{(h)}\left(N^{(h)}, Q^{(h)}\right)=\sum_{n=0}^{N^{(h)}} P_{\operatorname{loss}}^{(h)}\left(n, Q^{(h)}\right) v_{n}^{N^{(h)}}$

The quantities within the sum in (14) have been defined in (4) and (12) or (13) depending on which method is used. If 'method $A$ ' is used, the value of $\mathrm{P}_{\text {loss }}^{(\mathrm{h})}\left(\mathrm{n}, \mathrm{Q}^{(\mathrm{h})}\right)$ has to be taken from (12), while it has to be selected from (13) if 'method B' is chosen. (12) and (13) do not always represent the same quantity, but in all the cases considered in the simulations and in most of the situations of interest they originate very similar values of (14). This behaviour is due to the fact that, in these cases, there is an $\tilde{n}$, such that $\mathrm{f}_{\mathrm{j}}(\mathrm{n})<<1 \forall \mathrm{j}>1$ for a generic $\mathrm{n}<\tilde{\mathrm{n}}$ (condition that, as already stated, makes (12) and (13) equal) or $v_{n}^{N} \ll 1$ for $n \geq \tilde{n}$; in this last case (12) and (13) can be different but their values do not substantially affect the value of (14). Note that $n$ is a generic threshold value of $n$, used to clarify the description.

The introduction of this new approximation aims at simplifying the computational problems for a possible real implementation: the more internal sum in (12) can be critical from the computational point of view because the limit $n^{(h)}$ of the sum could be very large; on the contrary, for short buffer lengths, (13) has less terms in the inner sum and, then, requires significantly less time to be computed. The two approaches may be similar for very large buffers.

The use of the stationary distributions $\pi_{i}^{(h)}\left(n^{(h)}\right), i=0,1, \ldots, Q^{(h)}$ and $v_{n}^{N^{(h)}}$, $n=0, \ldots, N^{(h)}$, instead of the joint stationary distribution of the number of cells in the buffer and the number of active calls, is suggested by the large difference in the time scales between the cell and the active connections dynamics. More in detail, due to the previous assumption, the steady state values (7) can be supposed to be a good approximation of the real cell loss probability between two consecutive instants where the number $n^{(h)}$ of active connections changes. This kind of approximation, although in a different context, has been introduced in (Ghani, 1994), where its validity is also carefully analysed. The aim in this paper is to evaluate the approximation of the cell loss probability with $\mathrm{N}^{(\mathrm{h})}$ connections in progress, given by (14), in the two cases approached, namely, 'method A' (12) and 'method B' (13), on some specific examples by simulation. 


\section{COMPARISON WITH SIMULATION RESULTS}

In this Section, some simulation results are reported to evaluate the accuracy which can be achieved in the cell loss probability computation by using the methods presented in Section 3. Six different traffic classes have been used in the simulations, whose parameters are reported in Table 1. The first three classes are the same ones used in (Yang, 1995), to give the opportunity to compare our results with those reported therein. The simulations have been performed on SUN SparcStations 10 and 20 . The criterion used to stop the simulations is that the width of the $95 \%$ confidence interval be less then $10 \%$ of the estimated cell loss probability. Let us recall again that $P^{(h)}$ is the peak bandwidth, $B^{(h)}$ is the average burst length, $D^{(h)}$ is the average bandwidth for each connection of the generic traffic class (h).

Table 1. Traffic class characteristics.

\begin{tabular}{cccc}
\hline $\begin{array}{c}\mathrm{h} \\
\text { Traffic Class }\end{array}$ & $\begin{array}{c}\mathrm{P}^{(\mathrm{h})} \\
{[\text { Mbits/s] }}\end{array}$ & $\begin{array}{c}\mathrm{D}^{(\mathrm{h})} \\
{[\text { Mbits/s] }}\end{array}$ & $\begin{array}{c}\mathrm{B}^{(\mathrm{h})} \\
{[\text { Cells] }}\end{array}$ \\
\hline 1 (Voice) & 0.064 & 0.022 & 58 \\
2 (Data) & 10 & 1 & 339 \\
3 (Image) & 2 & 0.087 & 2604 \\
4 & 1 & 0.5 & 100 \\
5 & 2 & 0.4 & 500 \\
6 & 10 & 1 & 1000 \\
\hline
\end{tabular}

All the diagrams report the cell loss probability which has been computed by means of (14), in both cases 'method A' and 'method B', and by simulation, using an independent Talkspurt-Silence source traffic generator for each connection in the system.

The results have been so structured: in the first three figures (Figs. 4-6) the cell loss probability has been depicted versus the number of connections in progress for voice, data and image calls, respectively. In this test the buffer length is constant. The following three figures (Figs. 7-9) show the opposite situation: the number of connections in progress is constant and the buffer length varies. Figs. 7-9 are associated to voice, data and image calls, respectively. Figure 10 and Figure 11 show the same quantities as in Figure 7 and Figure 8, with a different service time. Concerning the other traffic classes (traffic classes 4,5 and 6), the cell loss probability is drawn vs. the buffer length, with a constant value of the number of connections in progress in Figs. 12-14, respectively. 
It is important to note that the behaviour of the cell loss probability presented in (14), in both cases of 'method A' and 'method B', does not have the limitations of cell-scale models of burst-scale models (Roberts, 1991), but the behaviour, even if approximated, is the same as in the simulations. The values of 'method B' are always a little lower than the values of 'method $A$ ', so that the approximation $B$ is better when the results are conservative. However, it is important to observe that the two approximations are almost overlapped in any situation considered.

Concerning the specific traffic classes, the current evaluation is really well suited for voice calls. In all graphs reported (Figure 4, 8 and 11) the results are very accurate and always conservative. So, the values may be regarded both as a good approximation and as an upper bound of the real behaviour. As far as data traffic is concerned, the results are very close to the simulative values (Figure 5, 7 and 10) but they are not always conservative (Figure 5 and 10).

The approximation is very good for traffic class 5 (Figure 13), conservative for traffic class 4 (Figure 12), except for very small values of buffer length, while it does not provide conservative results for traffic class 6 , even if the approximation may be considered good.

Particular attention has to be dedicated to image traffic. Even though the results in this context may seem quite good (Figure 6 and 9), it is needed to remember that also the reference model (MMDP) is not so suited for video traffic (even if video retrieval may be a special case). So, these results would probably require a deeper analysis before concluding about the approximation.

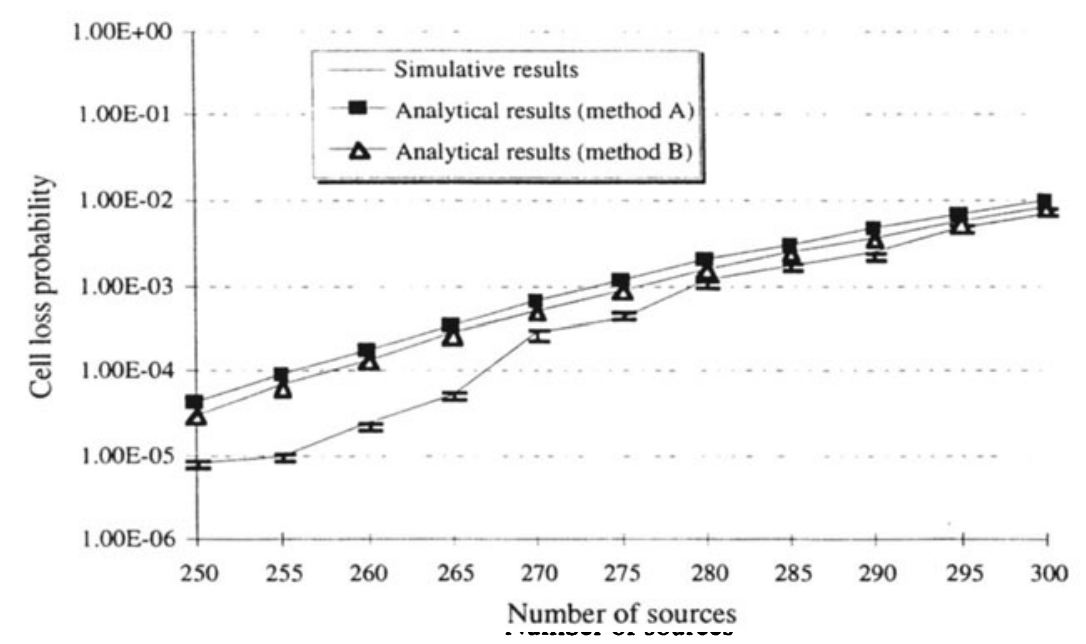

Figure 4. Cell loss probability versus the number of sources, $h=1$ (voice), $C=7$ $\mathrm{Mbits} / \mathrm{s}$, and $\mathrm{Q}^{(1)}=50$. 


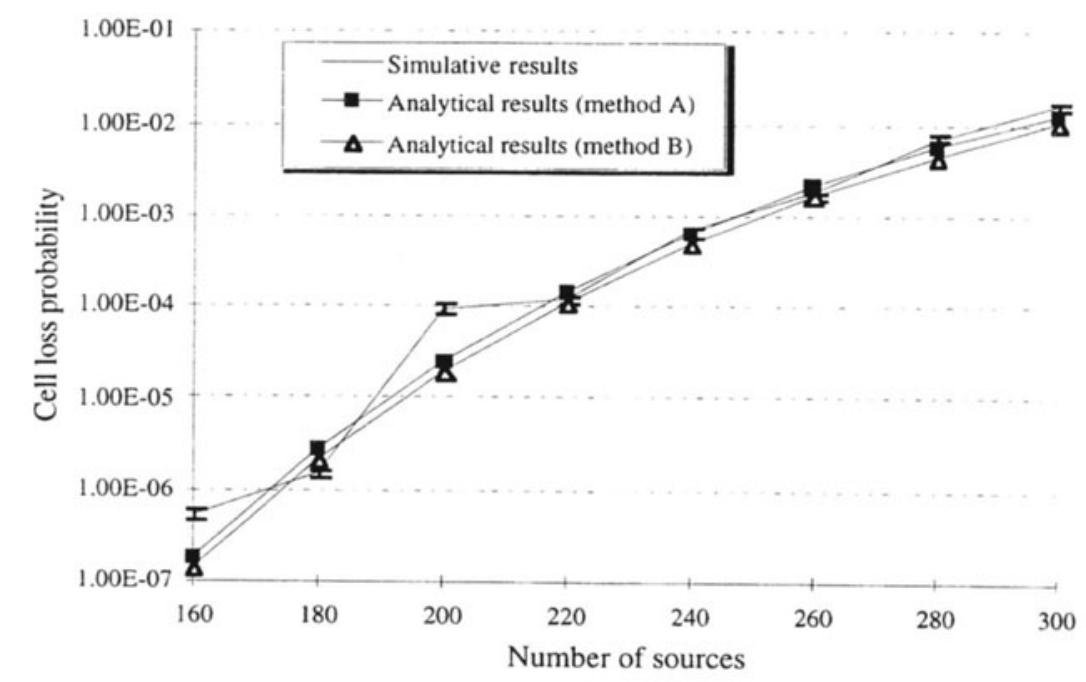

Figure 5 Cell loss probability versus the number of sources, $h=2$ (data), $C=350$ $\mathrm{Mbits} / \mathrm{s}$, and $\mathrm{Q}^{(2)}=50$.

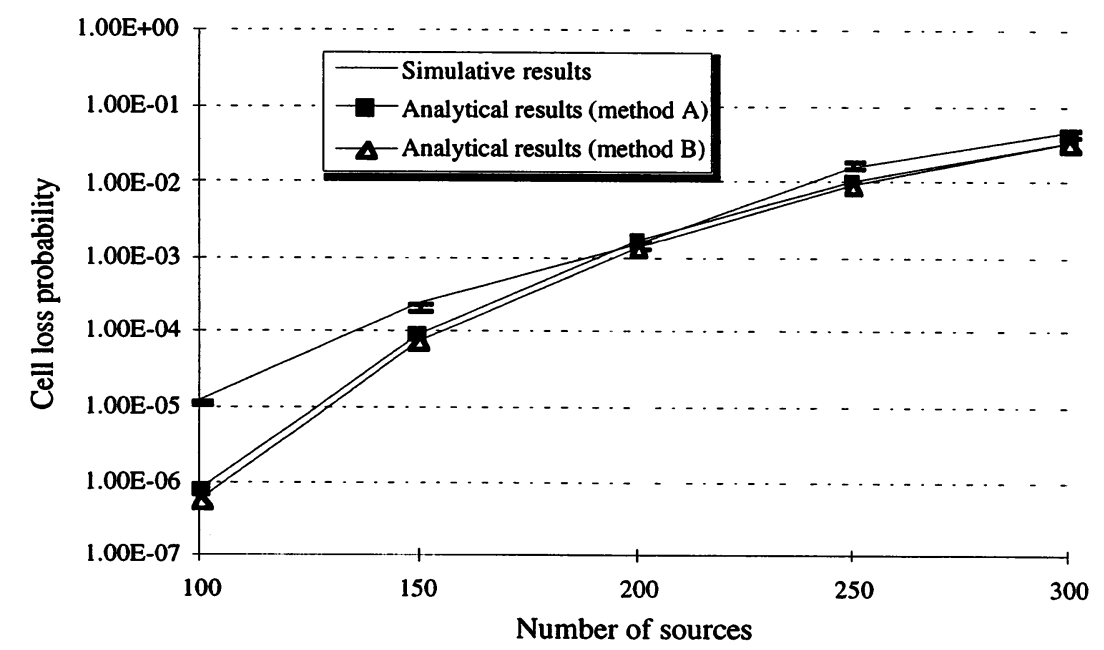

Figure 6. Cell loss probability versus the number of sources, $h=3$ (image), $C=30$ $\mathrm{Mbits} / \mathrm{s}$, and $\mathrm{Q}^{(3)}=50$. 


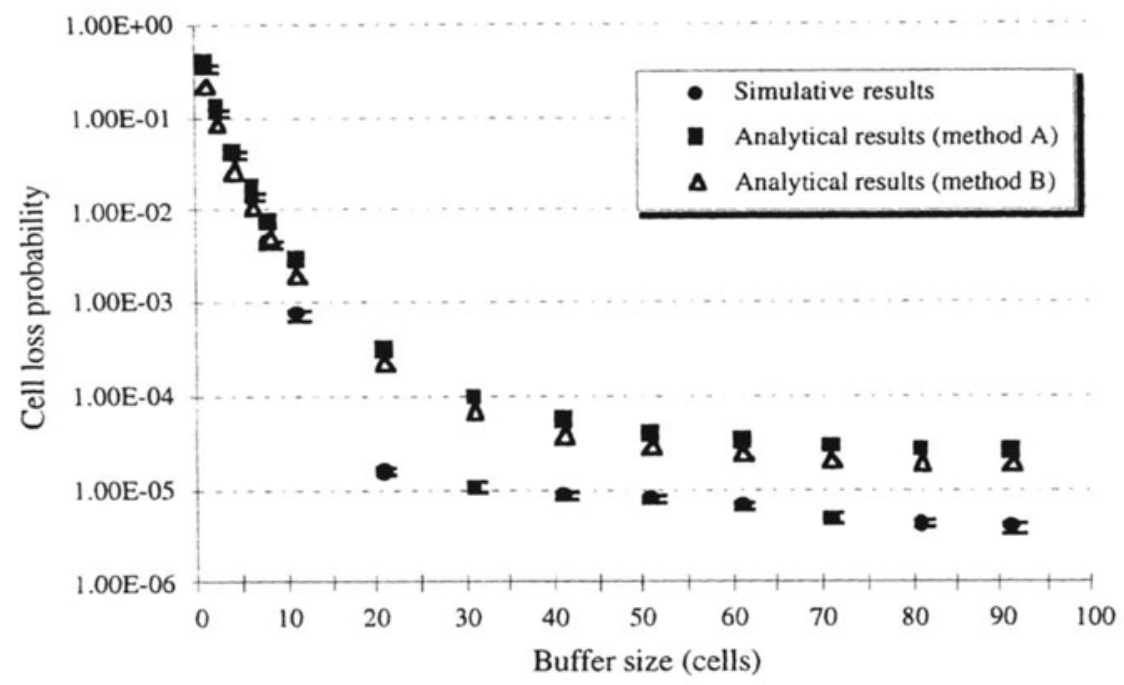

Figure 7. Cell loss probability versus the buffer size in cells, $h=1$ (voice), $C=7$ $\mathrm{Mbits} / \mathrm{s}$, and $\mathrm{N}^{(1)}=250$.

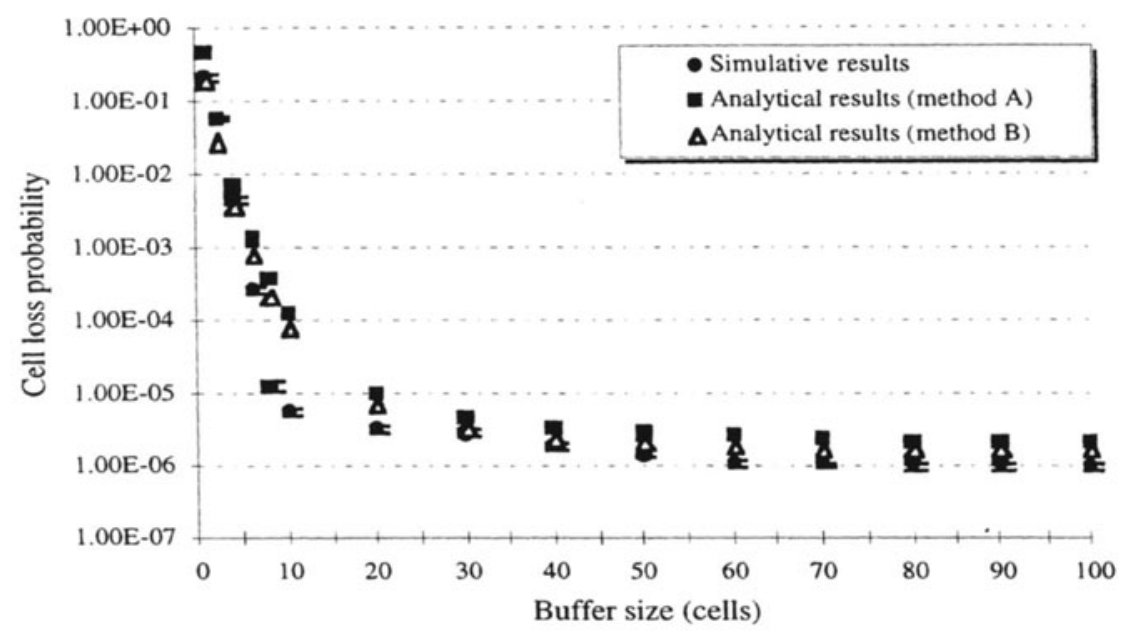

Figure 8. Cell loss probability versus the buffer size in cells, $h=2$ (data), $C=350$ $\mathrm{Mbits} / \mathrm{s}$, and $\mathrm{N}(2)=180$. 


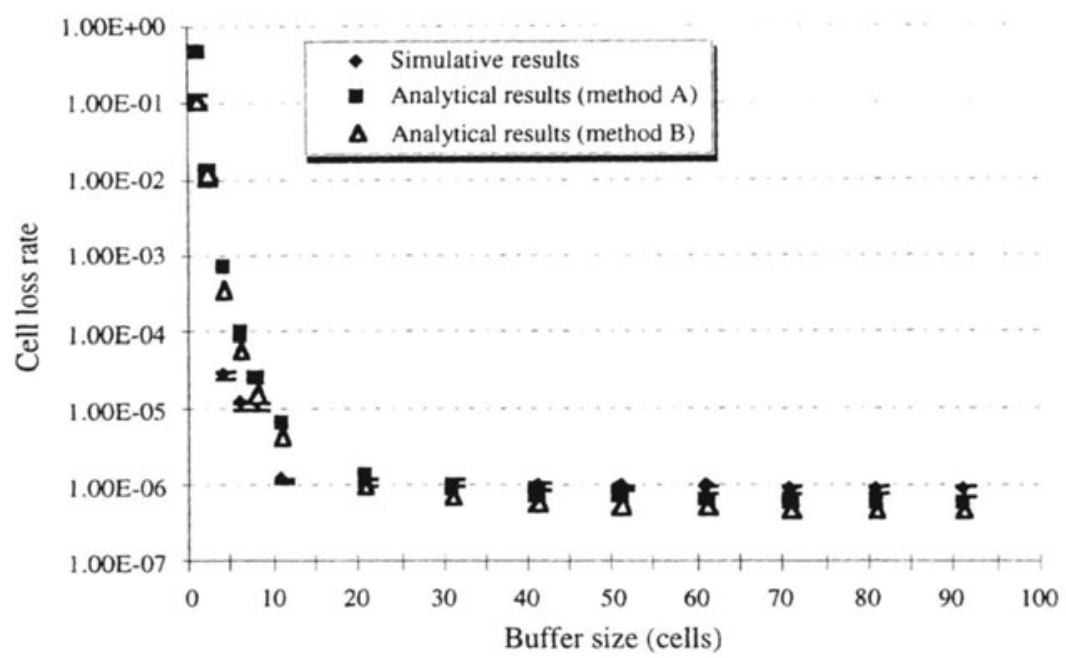

Figure 9. Cell loss probability versus the buffer size (cells), $h=3$ (image), $C=30$ Mbits $/ \mathrm{s}$, and $\mathrm{N}(3)=100$.

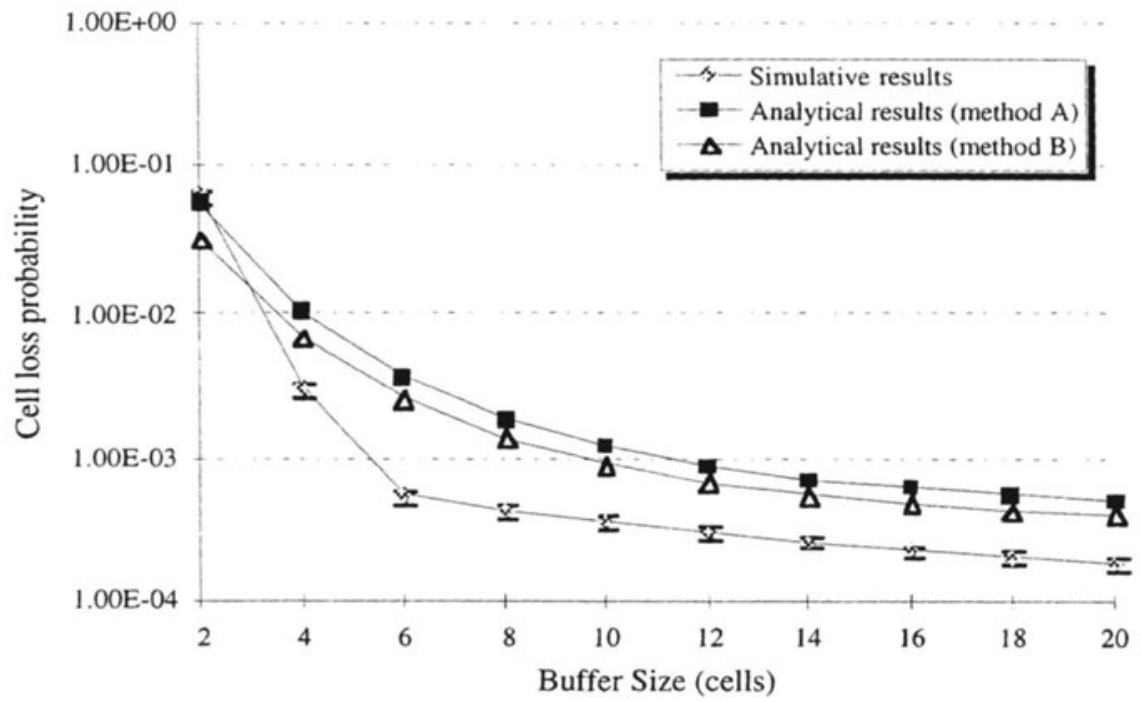

Figure 10. Cell loss probability versus the buffer size (cells), $h=1$ (voice), $C=0.7$ Mbits/s, and $\mathrm{N}(1)=17$. 


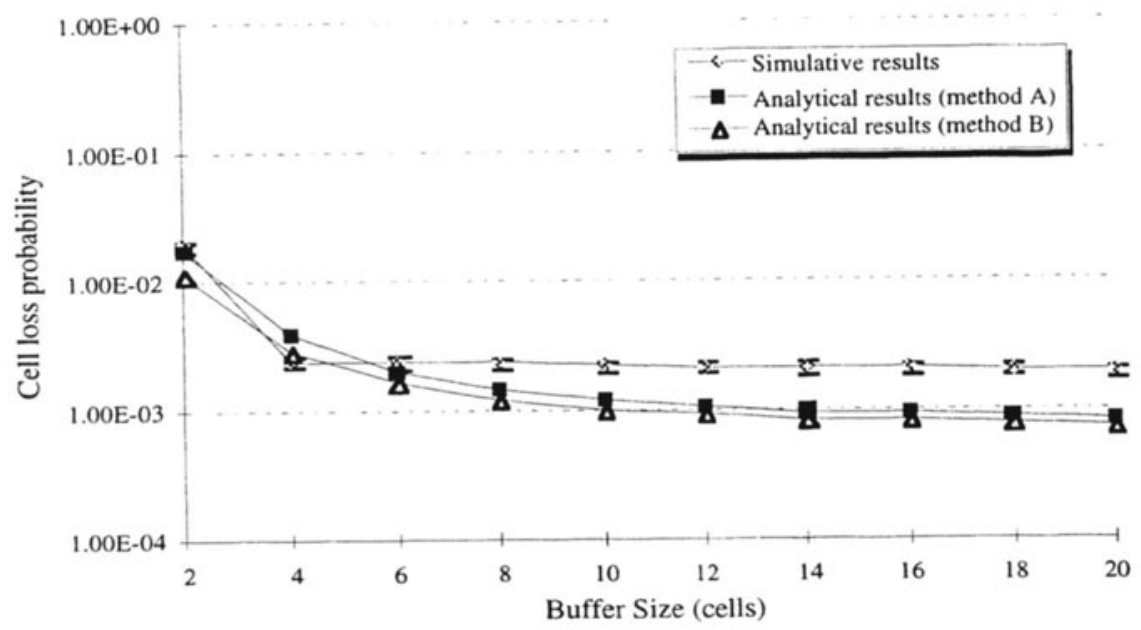

Figure 11. Cell loss probability versus the buffer size (cells), $\mathrm{h}=2$ (data), $\mathrm{C}=52$ $\mathrm{Mbits} / \mathrm{s}$, and $\mathrm{N}^{(2)}=17$.

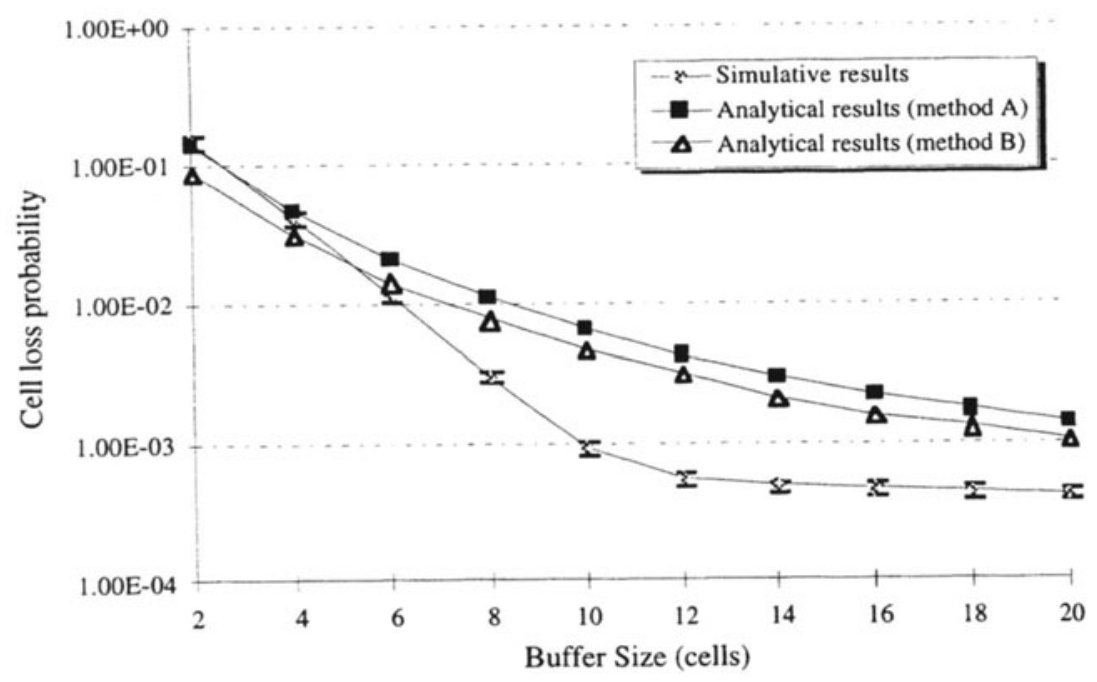

Figure 12. Cell loss probability versus the buffer size(cells), $h=4, C=50 \mathrm{Mbits} / \mathrm{s}$, and $\mathrm{N}^{(4)}=80$. 


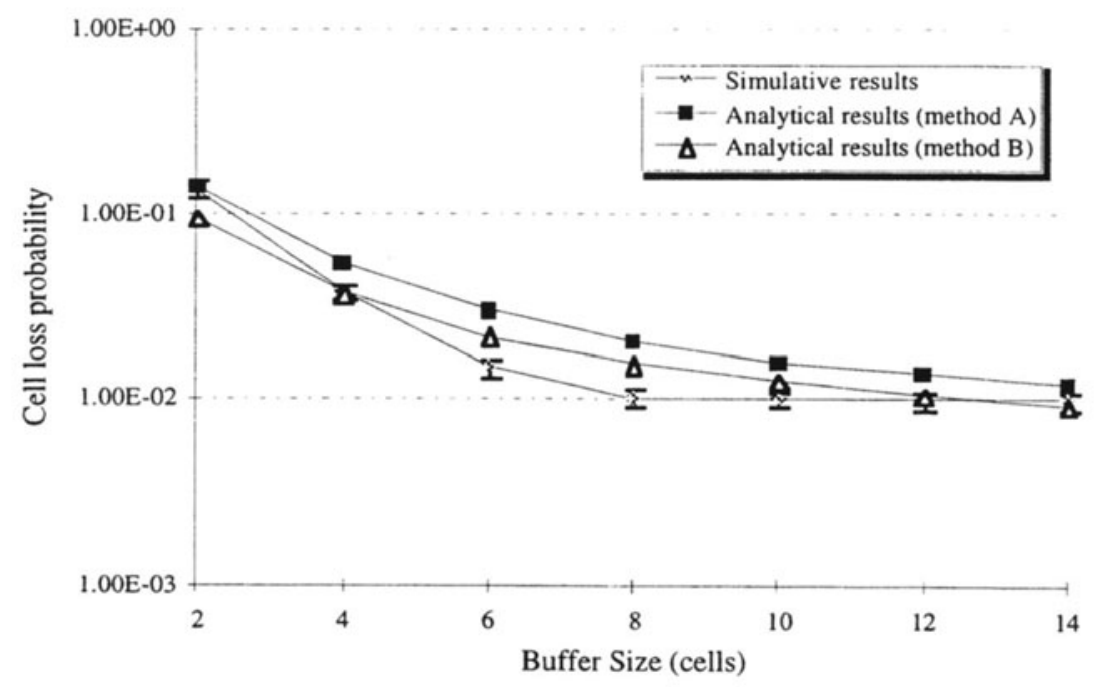

Figure 13. Cell loss probability versus the buffer size (cells), $h=5, C=50 \mathrm{Mbits} / \mathrm{s}$, and $\mathrm{N}^{(5)}=100$.

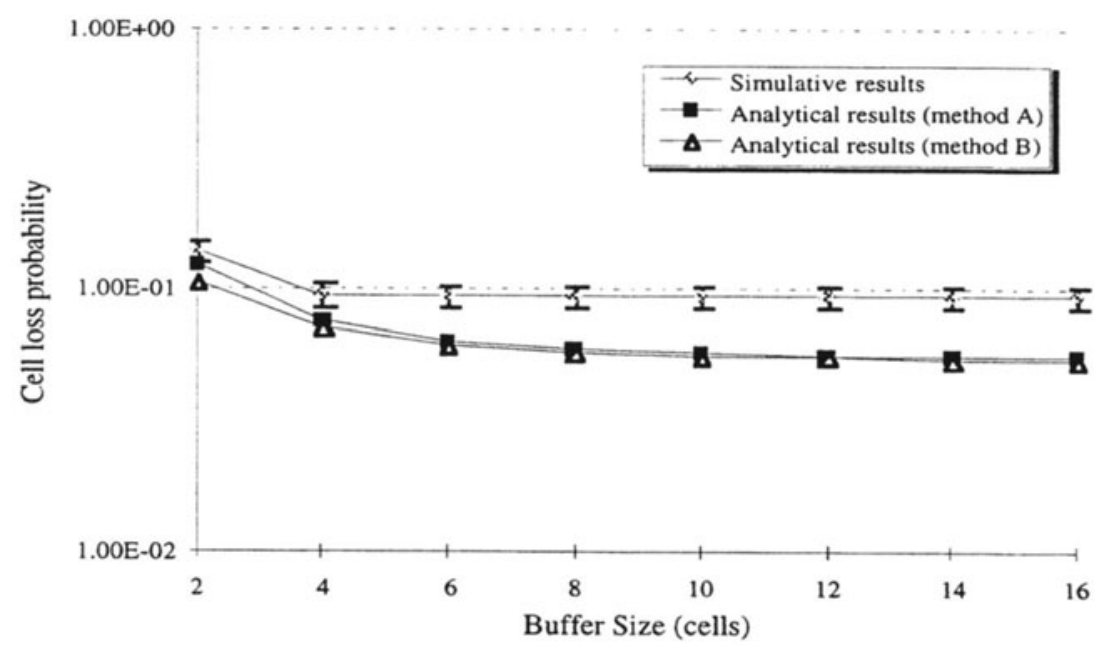

Figure 14. Cell loss probability versus the buffer size (cells), $h=6, C=50 \mathrm{Mbits} / \mathrm{s}$, and $\mathrm{N}^{(6)}=40$. 


\section{CONCLUSIONS}

A new approach to evaluate the cell loss probability in an ATM multiplexer has been presented in this paper. The traffic is segregated into classes. Each class has a dedicated buffer with constant service rate and it can be considered separately from the others. Each call of a specific traffic class is modelled by an IBP process and the superposition of these sources is considered, in order to evaluate the cell loss probability. The computed values are compared with simulation results, obtained by using the superposition of MMDP sources as traffic generator within the simulator. The model results particularly well suited for voice calls. Concerning data traffic, the approximation is very accurate but the results cannot be regarded as an upper bound for the actual call loss probability, because they are not always conservative. The video traffic would need further investigation, because even the model used here as a reference (MMDP) has been demonstrated to be not so suited for video traffic. In this last case the results presented should be considered just as examples. The new estimation is aimed at minimising the computational load for short buffer lengths in possible real implementation; the precise definition of the scope of the estimation would be necessary, in order to evaluate and possibly correct the potential problems about the not always conservative behaviour.

Some future research could be dedicated to further investigation to clarify whether the difference observed between the simulation results and the calculated quantities are introduced either by the approximation of the Talkspurt-Silence source model by an IBP arrival model, or by the decomposition approximation for computing the cell loss probability.

\section{REFERENCES}

Baiocchi, A. (1993), Accurate formulae for the loss probability evaluation in a large class of queueing systems. Performance Evaluation, 18, 125-132.

Bolla, R., Davoli, F., Lombardo, A., Palazzo, S. and Panno, D. (1994), Hierarchical dynamic control of multiple traffic classes in ATM networks. Europ. Trans. Telecommun., vol. 5, no. 6, 747-755.

Bolla, R. Davoli, F. and Marchese, M. (1995), Quality of Service management and control of multimedia applications: a scenario and two simple strategies. Proc. 20th IEEE Local Computer Networks Conference (LCN'95), Minneapolis, MN, 336-341.

Chao, H.J. and Uzun, N. (1995), An ATM queue manager handling multiple delay and loss priorities. IEEE /ACM Trans. Networking., vol. 3, no. 6, 652-659.

Cheng, T.H., Cheah, K.L., Gunawan, E., Oh, S.H. and Smith, D.G. (1996), A moment matching approach for modelling ATM cell arrival processes. International Journal of Communication Systems, 9, 1-3.

Choudhury, G.L., Leung, K.K. and Whitt, W. (1995), An inversion algorithm to compute blocking probabilities in loss networks with state-dependent rates. IEEE /ACM Trans. Networking., vol. 3. no. 5, 585-601. 
Cosmas, J.P. et al. (1994), A review of voice, data and video traffic models for ATM. Europ. Trans. Telecommun., vol. 5, no. 2, 11-26.

D'Ambrosio, M. and Melen, R. (1995), Evaluating the limit behavior of the ATM traffic within a network. IEEE /ACM Trans. Networking., vol. 3, no. 6, 832841.

Elwalid, A.I., Heyman, D.P., Lakshman, T.V., Mitra, D. and Weiss, A. (1995), Source models for VBR broadcast-video traffic. IEEE /ACM Trans. Networking., vol. 4. no. 1, 40-48.

Gagnaire, M., Kofman, D. and Korezioglu, H. (1995), An analytical description of the packet trains model for LAN traffic characterisation, in Performance Modeling and Evaluation of ATM Networks, (D.D.Kouvatsos, Ed.), 3-13.

Ghani, S. and Schwartz, M. (1994), A decomposition approximation for the analysis of voice/data integration. IEEE Trans. Commun., vol. 42, 2441-2452.

Gravey, A. and Hébuterne, G. (1992), Simultaneity in discrete-time single server queues with Bernoulli inputs. Performance Evaluation, vol. 14, no. 1, 123-131.

Heffes, H. and Lucantoni, D.M. (1986), A Markov modulated characterization of packetized voice and data traffic and related statistical multiplexer performance. IEEE J. Select. Areas Commun., vol. 4, no. 6, 856-867.

Herrmann, C. (1995), VBR Video in ATM without frame buffering: influence of a periodic correlation function on QoS parameters, in Performance Modeling and Evaluation of ATM Networks, (D.D. Kouvatsos, Ed.), 14-31.

Heyman, D.P. and Lakshman, T.V. (1996) Source models for VBR broadcast-video traffic. IEEE/ACM Trans. Networking, vol. 4, no. 1, 40-48.

Hui, J. Y. (1990), Switching and Traffic Theory for Integrated Broadband Networks. Kluwer Academic Publishers, Boston.

Kamitake, T. and Suda, T. (1989), Evaluation of an admission control scheme for an ATM network considering fluctuations in cell loss rate. Proc. IEEE Globecom '89, Dallas, TX, 1774-1780.

Kanakia, H., Mishra, P.P. and Reibman, A.R. (1995), An adaptive congestion control scheme for real time packet video transport. IEEE /ACM Trans. Networking., vol. 3, no. 6, 671-682.

Karlsson, G. (1995), Asynchronous transfer of video. Swedish Institute of Computer Science (SICS) Research Report, R95:14, 1-30.

Lee, D.S. and Sengupta, B. (1994), Temporal placement control of video frames in ATM networks. International Journal of Communication Systems, 7, 329-335.

Nagarajan, R., Kurose, J.F. and Towsley, D. (1991), Approximation techniques for computing packet loss in finite-buffered voice multiplexer. IEEE J. Select. Areas Commun., vol. 9, 388-393.

Onvural, R.O. (1994), Asynchronous Transfer Mode Networks: Performance Issues. Artech House, Inc., Norwood, MA.

Roberts J.W. (Ed.), (1992) COST 224 - Performance Evaluation and Design of Multiservice Networks. Commission of the European Communities, Brussels.

Roberts, J.W. and Gravey, A. (1991), Recent results on B-ISDN/ATM traffic modelling and performance analysis - A review of ITC 13 papers. Proc. IEEE Globecom '91, 1325-1330. 
Ross, K.W. (1995), Multiservice Loss Models for Broadband Telecommunication Networks. Springer, London.

Saito, H. (1994), Teletraffic Technologies in ATM Networks. Artech House, Inc., Norwood, MA.

Sohraby, K. (1993), On the theory of general ON-OFF sources with applications in high speed networks. Proc. Infocom '93, San Francisco, CA, 401-410.

Sriram, K. and Whitt, W. (1986), Characterizing superposition arrival processes in packet multiplexers for voice and data. IEEE J. Select. Areas Commun., vol. 4 , no. 6, 833-846.

Yang, T. and Tsang, H.K. (1995), A novel approach to estimating the cell loss probability in an ATM multiplexer loaded with homogeneous on-off sources. IEEE Trans. Commun., vol. 43, no. 1, 117-126.

\section{BIOGRAPHIES}

Raffaele Bolla was born in Savona, Italy, in 1963. He received the "laurea" degree in Electronic Engineering from the University of Genoa in 1989 and the Ph.D. degree in Telecommunications at the Department of Communications, Computer and Systems Science (DIST) of the University of Genoa, in 1994. From 1994 to 1996 he was a post-doc research fellow at DIST. Since November 1996 he is Assistant Professor in the same department. His current research interests are in management and control of STM and ATM networks, multimedia communications and multiple access in integrated mobile radio networks.

Franco Davoli was born in Genoa, Italy, in 1949. He received the "laurea" degree in Electronic Engineering from the University of Genoa in 1975. Since 1985 he has been Associate Professor and since 1990 Full Professor of Telecommunication Networks at the University of Genoa, where he is with the Department of Communications, Computer and Systems Science (DIST). From 1989 to 1991 and from 1994 to 1996, he was also with the University of Parma, Italy, where he taught a class in Telecommunication Networks, by means of interactive distance learning over ISDN. His current research interests are in bandwidth allocation, admission control and routing in STM and ATM networks, multimedia communications and services, and integrated services mobile radio networks.

Mario Marchese was born in Genoa, Italy, in 1967. He received the "laurea" degree in Electronic Engineering from the University of Genoa, Genoa, Italy, in 1992. He has concluded his Ph.D. in Telecommunications at the Department of Communications, Computer and Systems Science (DIST), University of Genoa. His research interests include traffic modelling, admission control and routing in ATMbased networks. 